\title{
Review of: "Single-nucleus transcriptomic analyses reveal microglial activation underlying cerebellar degeneration in Ataxia Telangiectasia"
}

\author{
Martin Lavin ${ }^{1}$ \\ 1 University of Queensland
}

Potential competing interests: The author(s) declared that no potential competing interests exist.

Progressive cerebellar degeneration is a major feature of the multi-system disorder ataxia-telangiectasia $(A-T)$. While the role of the protein defective in A-T, ATM, is well described in protecting the genome against DNA damage and in reducing the threat of oxidative stress, how it protects against the loss of Purkinje and granule neurons and the consequent neurodegeneration remains unresolved. An explanation for this dilemma is provided by Lai et al who employed single nucleus transcriptomic analyses of human cerebellum to reveal activation of inflammatory pathways in microglia and downregulation of calcium ion homeostatic pathways in Purkinje neurons. They interpret these data as a novel role for microglia activation in degeneration in A-T but this is not a new concept and indeed there is mounting evidence for a role for microglial in the pathogenesis of A-T (Ferro et al Trends in Neurosciences ,2019).While animal models of A-T do not re-capitulate the cerebellar ataxia characteristic of A-T more recently rat models have been shown to exhibit some degenerate changes in the nervous system. These studies demonstrate that unrepaired damage to DNA , leading to significant levels of cytosolic DNA in neurons and microglia, accompanied by activation of the cGAS-STING pathway and cytokine production, increases the inflammatory microenvironment which leads to dysfunction and death of neurons (Quek et al Hum Mol Genet, 2017). Microglial activation was observed in the cerebellum and spinal cord and progressed with disease phenotype suggesting that microglia activation was a key part of neurodegenerative changes in these rats. Furthermore, Song et al J Neuroscience 2019 reported a significant role for DNA damage, STING activation and induction of the innate immune system in the process of neurodegeneration in Atmdeficient mice. They showed that ATM-deficient microglia are hyperactivated to trigger a proinflammatory response and cause neurotoxicity. Reference is only made to the latter study here. While the findings in the present report are exciting it is important that these results be viewed more critically in the context of what is already known about microglial activation and neurodegeneration in A-T model systems. That said the approach described here is an exciting one that makes an important step forward in addressing neurodegeneration in A-T. It takes advantage of access to human post-mortem cerebellar vermis tissue using single nucleus RNA sequencing (snRNA-seq) to investigate individual cell and regionspecific alterations in A-T. The study is carefully carried out ensuring the diagnosis of A-T before going on to identify the major cell types in the cerebellum. It is of interest that expression of ATM was highest in 
microglia and that the expression of this protein and other DNA repair proteins was relatively reduced in murine microglia. This may well provide an explanation as to why the neurological phenotype is milder in A-T animal models. Cell type differential gene expression was most evident in microglia and Purkinje neurons, consistent with the reported roles for these cells in the neurodegenerative phenotype in A-T. An important observation is the upregulation of apoptosis signaling pathways in both Purkinje and granule neurons for A-T. However, an intriguing result is that the genes upregulated in Purkinje cells respond to extrinsic apoptotic signals while those in granule neurons are involved in intrinsic apoptotic signaling and response to DNA damage. This suggests that their model (Fig S8D) combining Purkinje and granule cell death in responding to neurotoxic cytokine effects needs more detail. What are the different forms of cell killing for the two cell types ? Does signaling emanating from the cell membrane apply to Purkinje cells only? Many reports over the years describe signal defects in A-T that occur independent of DNA damage (e.g. B and T-cell signaling, response of A-T cells to growth factors). Their observations that abnormal $\mathrm{Ca} 2+$ homeostasis may be a direct consequence of ATM loss of function in Purkinje neurons is relevant to this signaling defect. The suggestion that ATM may be a direct upstream activator of $\mathrm{Ca} 2+$ signaling is also attractive and some insight as to how this may relate to microglial activation and cell death is also worthy of consideration. Recent results that endoplasmic reticulum (ER)-mitochondrial connectivity through IP3R1GRP75-VDAC1, to maintain $\mathrm{Ca}^{2+}$ homeostasis in response to nutrient stress, can account for at least part of the mitochondrial dysfunction observed in A-T cells may be related to this (Yeo et al, iScience ,2020).In summary while this report is not novel in describing microglial activation and its implications for neurodegeneration in $\mathrm{A}-\mathrm{T}$, it provides individual cell resolution and identification of transcripts that further supports such a role, this time in A-T patients. It represents a very useful database to investigate genes and pathways that play important roles in protecting against neurodegeneration more generally. As pointed out above it falls somewhat short in addressing existing knowledge in this area of research and its value would be improved by more careful reading of the literature and inclusion of that data. Altogether a very valuable asset to investigate cell behaviour and cell -cell interactions in the cerebellum to maintain brain homeostasis. 